\title{
Ranking the Criteria of Quality Evaluation for Intellectual Information on Internet
}

\author{
Sarabjeet Singh Bedi ${ }^{1}$ and Amanjot Kaur ${ }^{2}$ \\ ${ }^{1}$ Department of Computer Engineering, M.J.P. Rohilkhand University, Bareilly, India \\ ${ }^{2}$ G. G. I. College, Bareilly, India
}

\begin{abstract}
Recent past has witnessed the fast use of the information and communication technology in the ever developing field of education. Enormous information is brought to the classroom for easy access by the learner anywhere in the world. Learners of today are distinguished by their proficiency in use of technology to know about assessment and successful management of informational opportunities. Critical analysis of existing education scenario emphasizes the introduction of major thrust areas as Information Communication Technology, literacy, organization of curriculum, and others. This is to elevate the standard of education and disseminate the information in shortest time with use of online and offline resources through internet. These are for professional growth, teaching support materials and other classroom related activities. The research work and true knowledge of topics calls for the need to evaluate the information provided on the internet. The present paper caters to this need, especially in context to intellectual information available on the internet. The research work in this paper is accomplished by selecting a system of quality criteria and defining in terms of internet usage for intellectual purpose. Several questions are designed to assess each criterion and as a result an instrument is developed and validated in the form of a questionnaire. The quality criteria taken into consideration are as Content, Objective reasoning, Scope, Authority; these are further classified into indicators for easy assessment. The collected data has been tabulated and analysed to rank the criteria as per their preference for quality assessment of the intellectual information available on the internet. The results indicate that authority is the most preferred criteria while objective reasoning is the least preferred one as per their calculated mean values.
\end{abstract}

\section{KEYWORDS}

Criteria Indicators; Intellectual Information; Internet; Quality Assessment;

\section{INTRODUCTION}

Advances in scientific research have given rise to sophisticated new technologies in the fields of Information Communication Technology (ICT) that drastically change the concept of a largesized diversified world to a global village. In the new millennium, the information revolution in the communication sector is proliferating towards digitized communication channels in cyber space. The communication among people through innovative computer technologies is creating limitless opportunities for accessing all social learning and intelligence. The internet is a vast network that connects many independent networks spanning over various countries in the world. It links computers of many different types, sizes and operating systems, along with many people of those countries that use the internet to communicate. The one thing all these computers have in common is the use of the Transmission Control Protocol/Internet Protocol (TCP/IP), which allows computers of different types to communicate with each other. Efficient storage and retrieval of reliable information are of crucial importance to science medicine, education and technology. There is a repository of information comprising the tremendous technological 
advances in human capabilities to code, encode, record, reproduce and disseminate it on the internet referred to as "publication inflation".

With the rapid and recent development of communication system and digital technology, society has become more technology oriented. The past decades have witnessed the pervasive use of information and communication technology in education, business, government and health care sectors. The advent of the internet has enormously altered the way the information is located by the researchers. It has become very easy to publish information on the World Wide Web (WWW). People can express the viewpoints easily as there is no editorial board to evaluate the quality and facts of the published information. The rapid growth in the diversification and dissemination of information by electronic means has the effect on keen awareness of the concept of information explosion. As citizens of the world, there is need to be conversant with the technologies and leverage its use for better quality of daily life. The awareness of the implication of technologies needs to be introduced at early stages for same reasons.

The user, teachers and students surf the internet to find and locate the suitable information. These all need to evaluate the credibility of the information. Although the web is easy to use, yet many users do not look into the details of quality of information provided to them. This problem becomes even more critical when unlike other printed books, journals and articles; almost any kind of information can be put on the web by anyone with the knowledge of internet and the computer. All this calls in for the evaluation of the quality of the information available on the web. One must evaluate the information available on the internet to determine the accuracy of the limited viewpoints presented on the internet.

In the view of above mentioned issues especially in context to intellectual information available on the internet, this paper presents the quality criteria for evaluating intellectual information. The research work is accomplished by selecting a system of quality criteria and defining in terms of internet usage for intellectual purpose.

\section{TECHNOLOGY FOR INTELLECTUAL INFORMATION ON INTERNET}

Instructional technology is the key to the universal goal of quality education. Connick [1] described the quality to continuously increase rapidly in educational technology. This created a new educational culture with no intrinsic huddles to total quality assurance using instructional technology in higher education. All the problems were handled through better administration and planning.

Quality of online education is dealt with appropriateness and completeness to be adjudged by the faculty in typical academic culture for encouragement of course development autonomy. It was defined as evaluation of specific learning outcomes with help of the instructor formulating the syllabus. The quality standards assess the content of the objectives as the privilege of the faculty [2]. Students have easy access to support services like library, peer interaction, faculty members and online resources that certify and convince validity of quality standards.

A member of "Delphi Forums" provided the idea for evaluating the quality of information with the help of four key considerations viz. accuracy, authority, currency and timeliness [3]. Accuracy pertained to fact or opinion, clarity and generalizations of the author information while the authority related to the expertise and credibility of the author. Lastly the currency and timeliness check the date of publication for latest and advanced information as criteria for evaluation. 
International Journal on Integrating Technology in Education (IJITE) Vol.3, No.2, June 2014

A study was conducted in National Institute of Education at Singapore to determine the perception of trainee teachers towards internet to select effective sites and quality information for their teaching purpose [4]. The results were analysed as the respondents indicated that up to date information was an important criteria while the identity of the individual or group creating the website was considered the least important criteria to select an internet website for educational purpose.

As per the experiences, the population increase and knowledge explosion affected the pattern of life and education, as the population increase was equivalent to the new patters of knowledge that came into being [5]. There has been a rapid development of communication technology in education at all levels with a purpose to extend educational facilities and upgrade instruction. The new technologies as an enhancement to the traditional instruction assisted the teacher to do the work in efficient manner for achievement of educational objectives. The maximum output could be availed with good availability, acceptability, economy and validity of the media used for intellectual information.

\section{IDENTIFICATION OF QUALITY ASSESSMENT CRITERIA}

In order to identify the criteria of the evaluation system, a search was made for the principal evaluation models of printed and electronic resources. Later, a comparative analysis had been made to identify the criteria used more frequently. As a result of this analysis, it was found that the criteria for the evaluation of an electronic resource, it was necessary to fall back on the traditional criteria. The identification used for evaluating electronic academic resources proved more difficult. To identify these indicators, it was necessary to review all the criteria proposed by the different authors for evaluation of electronic resources, to rearrange the questions according to criteria obtained earlier, to eliminate similar questions and to restruct the indicators.

It deals with the identification of the quality criteria for evaluation of educational information in the media. The initial source for this phase was the study that listed 125 indicators of quality [6]. Also included in this phase was the compilation of 66 known evaluation tools for internet usage by others [7]. First the various criteria were defined and integrated as content, objective reasoning, scope, and authority. Later on the quality criteria indicators were also defined and integrated. Lastly, the questions were formulated for determination of presence or absence of each indicator. In the present study the quality of the educational information available on the internet has been assessed based on the criteria as Content, Objective reasoning, Scope, and Authority. These in turn ranked the quality of internet information with the help of the data collected, analysed and interpreted by the researcher.

\subsection{Content}

The Content is referred as the information available from any source. This criterion has indicators named as purpose, currency, uniqueness and intended audience that reveal the quality of information on internet.

\subsection{Objective Reasoning}

Objective Reasoning is the extent to which the information material expresses facts or information without distortion by personal feelings and other biases. The hypertext links also reveal the objectivity of information. It has become very difficult to determine the objectivity of web pages unless the purpose of the individual or group presenting the information is clearly stated. 


\subsection{Scope}

It refers to the sources included in the information presented on the internet. It presents the information as an implied area in introduction. The scope of the material intends to cover the area with in-depth knowledge as per the level of the readers [8]. The quality indicators to be taken under scope include breadth, depth, time and format of the information. The cost of internet usage is also a factor.

\subsection{Authority}

Authority is the extent to which material is the creation of person or organization recognized to have definitive knowledge of a given subject area. The author qualifications, publisher regulations, reviews about author product are indicators for assessment of authority of the information available on the internet.

\section{CONSTRUCTION OF ASSESSMENT TOOL}

After the identification of the different quality criteria, compilation with consulting a Subject Matter Expert (SME) and editing the listed quality criteria; a draft of the evaluation tool was developed for the teachers during this phase. The questionnaire was divided into two subparts. First part consisted of personal information of the respondent along with the internet usage by them for any purpose. It contained information regarding age, sex, internet access and their frequent usage by the respondent. The second part of questionnaire consisted of four main quality criteria as content, objective reasoning, scope, and authority. The researcher had further divided these quality criteria into various quality indicators that represented the whole criteria. The content and authority have six quality indicators in each, while objective reasoning and scope as quality criteria have only four indicators for the representation. Criteria wise distribution of items has been summarized in Table 1.

Table 1. Quality Criteria and Its Indicators.

\begin{tabular}{|l|l|}
\hline Quality Criteria & Quality Criteria Indicators \\
\hline Content & $\begin{array}{l}\text { Purpose, Currency, Hypertext Links, Uniqueness, } \\
\text { Copyright }\end{array}$ \\
\hline Objective Reasoning & Factual, Validity, Linkage, Fairness \\
\hline Scope & Breadth, Depth, Time, Format \\
\hline Authority & $\begin{array}{l}\text { Author Information, Organizational Authorship, Author } \\
\text { Credentials, Sources, Contact Information, Domain Name } \\
\text { (URL) }\end{array}$ \\
\hline
\end{tabular}




\section{RESULTS AND DISCUSSIONS}

The data collected from personal data schedule was tabulated for easy analysis of the study. Socio personal traits, internet accessibility, internet usage and the web sites most frequently visited by the respondent teachers for educational purpose were tabulated as simple frequencies and representative percentages. A total of 120 candidates participated as respondents in the study. The survey has been conducted in one of the metro city of North India.

For urban areas there was equal participation by male and female that denoted equality of education for all. The $80 \%$ respondents owned personal computer for easy internet access while $60 \%$ of them access the internet at the education institute itself. Many of the respondents surf the internet to access the information for education purpose. The respondents used the internet with various search engines like Google, Altavista, MSN on weekly basis to get updated about the latest information and events.

When sought for the basis for search, the values show that about three-fourth respondents search internet based on topic of the study for the assignment. The name of the author and his credentials were most taken care criteria for internet search. The results showed that most candidates found the information available on the internet to be useful to assign projects for educational purpose in classroom situations. The researcher has collected and tabulated the mean values of all the quality criteria. The formula for the mean $(\mathrm{M})$ of a series of ungrouped measures is given in (1) [9].

$$
M-\frac{2 x}{N}
$$

Where $\mathrm{N}$ is the number of measures in the series, $\mathrm{X}$ is a score.

This has been done to provide ranks to the various quality criteria based on mean values. Table II represents the corresponding values regarding preference to rank the educational information available on the internet by post graduate professional course students.

Table 2. Ranking of Quality Assessment Criteria.

\begin{tabular}{|l|l|l|}
\hline Quality Assessment Criteria & Mean Values obtained & Ranks ascertained \\
\hline Authority & 4.31 & I \\
\hline Content & 3.77 & II \\
\hline Objective Reasoning & 2.56 & III \\
\hline Scope & 2.20 & IV \\
\hline
\end{tabular}

The mean value for authority has been observed to be maximum as 4.31 . This has been considered the most preferred criteria for evaluation by the candidates. The content has been the next important criteria as per their preference with mean value of 3.77. The least preferred has been the scope with the calculated mean value of 2.20 showing least preference of candidates for the factual, valid, linked and fair information available on the internet.

Similar results have been obtained when a research survey was conducted at National institute of education at Singapore [4] to determine the perception of pre-service trainee teaches for selecting effective websites. The most preferred ones included the currency, ease of navigation and guide access to website. 
International Journal on Integrating Technology in Education (IJITE) Vol.3, No.2, June 2014

\section{CONCLUSION AND EDUCATIONAL IMPLICATIONS}

The information has been found to be complete on the various intellectual web pages. The scope of the intellectual information is quiet neglected by the authors publishing the web pages on the internet. The in-depth information is absent as only a brief account of the same is provided on the webpage.

The main purpose of the present study was to rank the quality assessment criteria for intellectual information available on the internet by comparing the observed mean values for the various quality criteria indicators. The assessment criteria included content, objective reasoning, scope and authority. It has been concluded that the candidates found authority and content of information to be more important than fairly objective and in-depth content of any intellectual web page.

The survey findings reveal that the educational program should be based on individual strength and weakness of the students. Material should be divided into interlinked small units for self paced learning. The teacher should provide an enriched environment to uplift the credibility standard of information on internet. For this, the teachers have to define the internet usage as a unique educational contribution.

The content should be evaluated for different indicators such as purpose, currency, hypertext links, uniqueness and copyright. Learning should be based on self-pacing with realistic deadlines given for the completion of assignment based on educational information available on internet. The teachers may contact the author for detailed information on the same topic to increase its reliability. The quality criteria selected in this study are not exhaustive. More criteria may be studied before a comprehensive view of educational information is obtained. Similar studies can be conducted for the different courses and different sectors of education (like government and private) for comparative analysis. The assessment of other type of information as institutional, religious, moral, environmental, marketing and medical may also be studied.

\section{REFERENCES}

[1] G. P. Connick, (1997) "Issues and trends to take us into the twenty first century", New Directions for Teaching and Learning, 71, pp 1-7.

[2] G. D. Garson, (1997) Academic Micro Computing: A resource guide, Sage Publications, Newbury Park.

[3] H. Walt, (2001) Producing Quality Web Page Content, Available: http://www.people.delphiforums.com

[4] M. S. Khine, (2004) "Finding quality Information on Internet for Educational Use", Staff and Educational Development International Journal, Vol. 8, No. 2, pp 109-116.

[5] N. Venkataiah, (2004) “An overview of Educational Technology”, Educational Technology, Vol. 1, No. 19.

[6] G. L. Wilkinson, L. Banett and K. Oliver, (1997) "Evaluation criteria and indicators of quality for internet resources", Educational Technology, Vol. 34, No. 3, pp 52-58.

[7] L. Gurak and L. Bobendrier L, (1999) "Net analysis of guidelines for web-based research", Available: http://www.isc.umn.dey.research/papers/Boweer-web.htm

[8] A. G. Smith, (1997) "Testing the surf: Criteria for Evaluating Internet Information Resources", The Public Access Computer Systems Review, Vol. 8, No. 3, pp 24-27.

[9] J. W. Best and J. V. Kahn, (2004) Research in Education, 7th Ed., Printice Hall India Pvt. Ltd., New Delhi. 
International Journal on Integrating Technology in Education (IJITE) Vol.3, No.2, June 2014

\section{Authors}

Sarabjeet Singh Bedi is presently working as senior faculty member in Department of Computer Science and Information Technology, MJP Rohilkhand University Bareilly, India. He is having 16 years of academic, administrative and research work experience. $\mathrm{He}$ has received Master of Engineering degree in Computer Science and Engineering with honours from NIT-TTR, Chandigarh. PhD degree has been awarded to him by Indian Institute of Information Technology, Gwalior, India in Information Technology. His research area includes Network Management and Security, Digital Image Processing. He has published several papers in reputed international/national journals including chapters in edited books. He has received institute medal from Thapar University, Punjab and merit certificate for best paper presentation at World Congress on Engineering, London, U.K, In July 2008.

Amanjot kaur did her M.Sc., M. Ed. and Ph.D. in Education from MJP Rohilkhand University, Bareilly. She is having 08 years school and graduate level teaching experience. Her research area is use of ICT in secondary and higher education. She has organized workshop and delivered expert lecture in the area of quality education. She has published several research papers in reputed international/national journals.
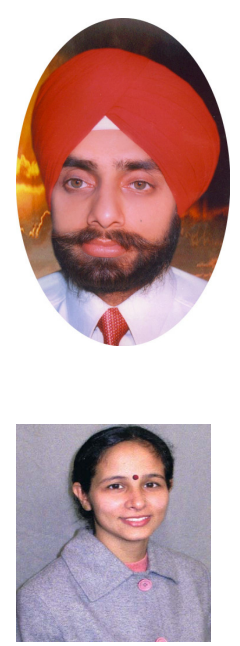
International Journal on Integrating Technology in Education (IJITE) Vol.3, No.2, June 2014

I NTENTIONAL BLANK 